\title{
Direct Estimation of Optical Parameters From Photoacoustic Time Series in Quantitative Photoacoustic Tomography
}

\author{
Aki Pulkkinen*, Ben T. Cox, Simon R. Arridge, Hwan Goh, Jari P. Kaipio, and Tanja Tarvainen
}

\begin{abstract}
Estimation of optical absorption and scattering of a target is an inverse problem associated with quantitative photoacoustic tomography. Conventionally, the problem is expressed as two folded. First, images of initial pressure distribution created by absorption of a light pulse are formed based on acoustic boundary measurements. Then, the optical properties are determined based on these photoacoustic images. The optical stage of the inverse problem can thus suffer from, for example, artefacts caused by the acoustic stage. These could be caused by imperfections in the acoustic measurement setting, of which an example is a limited view acoustic measurement geometry. In this work, the forward model of quantitative photoacoustic tomography is treated as a coupled acoustic and optical model and the inverse problem is solved by using a Bayesian approach. Spatial distribution of the optical properties of the imaged target are estimated directly from the photoacoustic time series in varying acoustic detection and optical illumination configurations. It is numerically demonstrated, that estimation of optical properties of the imaged target is feasible in limited view acoustic detection setting.
\end{abstract}

Index Terms - Bayesian methods, biomedical optical imaging, inverse problems, photoacoustic effects, tomography, ultrasonic imaging.

\section{INTRODUCTION}

Q UANTITATIVE photoacoustic tomography (QPAT) refers to the problem of forming quantitative images of the underlying material parameters of an imaged target based on boundary measurements of acoustic pressure waves generated by the photoacoustic effect. The propagation and subsequent absorption and thermalisation of a short light pulse

Manuscript received March 21, 2016; accepted June 13, 2016. Date of publication June 15, 2016; date of current version October 27, 2016. This work has been supported by the Academy of Finland (projects 136220, 272803, 286247, 250215 Finnish Centre of Excellence in Inverse Problems Research). Asterisk indicates corresponding author.

*A. Pulkkinen is with the Department of Applied Physics, University of Eastern Finland, 70211 Kuopio, Finland.

B. T. Cox is with the Department of Medical Physics and Biomedical Engineering, University College London, WC1E 6BT London, U.K.

S. R. Arridge is with the Department of Computer Science, University College London, WC1E 6BT London, U.K.

H. Goh is with the Department of Mathematics, University of Auckland, and Dodd-Walls Centre for Photonic and Quantum Technologies, Auckland 1142, New Zealand.

J. P. Kaipio is with the Department of Applied Physics, University of Eastern Finland, 70211 Kuopio, Finland, and also with the Department of Mathematics, University of Auckland, and Dodd-Walls Centre for Photonic and Quantum Technologies, Auckland 1142, New Zealand.

T. Tarvainen is with the Department of Applied Physics, University of Eastern Finland, 70211 Kuopio, Finland and also with the Department of Computer Science, University College London, WC1E 6BT London, U.K.

Color versions of one or more of the figures in this paper are available online at http://ieeexplore.iee.org.

Digital Object Identifier 10.1109/TMI.2016.2581211 in the imaged target induces, via the photoacoustic effect, an excess pressure, called the initial acoustic pressure distribution. For absorber sizes of a few millimeters or less, this initial pressure distribution relaxes by radiating as a broadband pulse of sound in the ultrasonic frequency range. This sound can be observed on the boundaries of the imaged target and it forms photoacoustic time series (i.e. the measurement data). Common parameters of interest to be estimated in QPAT include: the spatial distribution of the light absorbing chromophores or the optical absorption, the optical scattering, and the Grüneisen parameter linking the initial pressure distribution to the absorbed optical energy distributions.

QPAT can be seen as the next step after photoacoustic tomography (PAT). In PAT, images of the initial pressure distribution, also called the photoacoustic images, are of principal interest. In many situations these images reflect, for example, the anatomical information of the target as produced by its internal distribution of absorbing chromophores. For a review on PAT in general, see e.g. [1]-[4]. Common methods to solve the acoustic inverse initial value problem of PAT include back projection, time reversal, and model based inversion approaches [5]-[8]. Reconstruction methods in specific acoustic measurement geometries, such as plane, cylinder, sphere, and polyhedra have been devised [5], [9]-[15], as well as methods that operate in more generic acoustic measurement setting [16], [17]. In addition to acoustically homogeneous situations, the acoustic inverse initial value problem with heterogeneous speed of sound [18]-[23] and attenuating propagation of sound [24]-[26] have been studied. In acoustically limited view setting, the acoustic inverse problem in QPAT becomes ill-posed and the estimates of the initial pressure distribution suffer from artefacts [27]. To alleviate the issue, regularization approaches using prior information on the spatial distribution of the initial pressure [28], [29] and model based inversion with MoorePenrose pseudoinverse [30] have been used. Additionally, use of acoustic mirrors and scatterers can improve the estimation of the initial pressure distribution in acoustically limited view setting [31]-[34]. For a review on the mathematics of the acoustic inverse initial value problem in PAT, see e.g. [35].

The additional step that QPAT takes, the quantitation, is the estimation of the spatial distributions of the optical parameters that result in the formation of the initial pressure distribution [36]-[38]. Commonly, a model based inversion approach is used to solve the inverse problem in QPAT. Both, the radiative transfer equation (RTE) 
based optical propagation model [39]-[45] and its diffusion approximation (DA) [46]-[55] have been used. In addition to using photoacoustic images as the input data in the quantitation step, combined data formed by the photoacoustic images and diffuse optical tomography (DOT) measurement data have been investigated [42], [56], [57]. Use of measurements obtained with illuminations performed at multiple wavelengths (i.e. spectral measurements) in order to obtain estimates of the concentrations of specific chromophores based on their absorption spectra have been studied [58]-[63]. Within the DA framework, analytical reconstruction approaches have been devised [64], [65]. For mathematical analysis of the optical inverse problem in QPAT within the RTE and the DA frameworks, see e.g. [66], [67].

In addition to approaches where the initial pressure distribution is estimated first and the optical parameters of interest afterwards, direct estimation of the optical parameters from the photoacoustic time series has been performed recently. In [68], [69], the DA based optical framework was used to derive a linear Born approximation based model. The model was subsequently coupled with a homogeneous acoustic propagation model, and perturbations of optical absorption and diffusion parameters were estimated directly from the photoacoustic time series. In [70], the DA based Born approximation and the full non-linear DA was used to estimate the optical absorption, as well as the speed of sound of the imaged target. In [71], the absorption parameter was reconstructed within the nonlinear DA framework. In [72], the RTE based optical forward model was coupled with a homogeneous acoustic propagation model. The paper demonstrated improvement of the optical absorption estimates, when the estimates were formed directly from the photoacoustic time series. In all [70]-[72], scattering was assumed to be known. In [73], the studied photoacoustic illumination and acoustic detection setup was such that the acoustic inverse problem could not be solved in a stable fashion, and thus the inverse problem of QPAT required the use of a direct estimation approach.

In this paper, the inverse problem of estimating the optical parameters directly from the photoacoustic time series in QPAT is further investigated. Simultaneous estimation of both absorption and scattering, with parameter values corresponding to realistic values in soft biological tissues, is considered in two dimensional (2D) and three dimensional (3D) limited acoustic view situations with different optical illuminations. The inverse problem is described by using a Bayesian approach to ill-posed inverse problems [74]-[76]. In the Bayesian approach, the inverse problem is viewed in the framework of statistical inference. All parameters are treated as random variables which depend on each other through a model and information about these parameters is expressed by probability distributions. In quantitative tomography, point estimates of the posterior distributions of unknown parameters are determined based on the measurements, the model, and the prior information. Thus, instead of qualitative PAT images, in Bayesian approach to QPAT one is aiming at obtaining quantitative estimates of the parameters of (primary) interest and their uncertainties.
The forward model used in this paper is formed by combining an optical model with an acoustic propagation model. For the optical model, the DA is used. Green's function based solution to homogeneous acoustic wave equation is used for the acoustic model. The resulting forward model maps the optical parameters of the imaged medium to the photoacoustic time series on the boundary of the target.

The approach is tested with simulations. The effect of the acoustic measurement geometry, the effect of the noise level, and the effect of the optical illuminations used to generate the data are analysed.

\section{MOdeL}

The photoacoustic effect can be modelled by coupling the optical and acoustic propagation models. The difference in propagation speed of light and sound means that the initial pressure distribution can be regarded as instantaneous with respect to the acoustic model. This means that the coupling of the models can be achieved by simple composition.

\section{A. Optical Model}

In this work, the propagation of light is modelled using the DA. The DA and the used boundary condition describing the flunce $\Phi$ within the computation domain $\Omega \subset \mathbb{R}^{n}$ are [77]

$$
\begin{cases}-\nabla \cdot \kappa \nabla \Phi+\mu_{\mathrm{a}} \Phi=0, & r \in \Omega \\ \zeta_{n} \Phi+\frac{1}{2} A \kappa \nabla \Phi \cdot v=s, & r \in \partial \Omega\end{cases}
$$

where $\kappa=\left(n\left(\mu_{\mathrm{a}}+\mu_{\mathrm{s}}^{\prime}\right)\right)^{-1}$ is the optical diffusion, $\mu_{\mathrm{a}}=\mu_{\mathrm{a}}(r)$ is the optical absorption parameter, $\mu_{\mathrm{s}}^{\prime}=\mu_{\mathrm{s}}^{\prime}(r)$ is the optical reduced scattering parameter, $\zeta_{n}$ is a dimension dependent scaling factor $\left(\zeta_{2}=\pi^{-1}\right.$ and $\left.\zeta_{3}=0.25\right)$. Further, $A$ and $s=s(r)$ describe the light reflectivity and the inward light current (i.e. the light source) on the boundary $\partial \Omega$. Throughout this work, value $A=1$ is used for the reflectivity corresponding to matched refractive indices inside and outside $\Omega$.

Absorption of light creates spatial absorbed energy density field $H$ given by

$$
H\left(r ; \mu_{\mathrm{a}}, \mu_{\mathrm{s}}^{\prime}, s\right)=\mu_{\mathrm{a}}(r) \Phi\left(r ; \mu_{\mathrm{a}}, \mu_{\mathrm{s}}^{\prime}, s\right),
$$

where the parameters are as in (1).

In this work, the optical propagation model (1) is discretized using Galerkin finite element method (FEM) [78]. FEM discretization is performed in a regular grid composed of either square (in 2D) or cube (in 3D) elements. The optical properties $\mu_{\mathrm{a}}$ and $\mu_{\mathrm{s}}^{\prime}$, as well as the fluence $\Phi$ are discretized using $Q$ piecewise bilinear (in 2D) or trilinear (in 3D) base functions defined at grid nodes $r_{q} \in \mathbb{R}^{n}$, with $q=1, \ldots, Q$. Thus, the parameters and the fluence are approximated as

$$
\begin{aligned}
& \mu_{\mathrm{a}}(r) \approx \mu_{\mathrm{a}}^{h}(r)=\sum_{q=1}^{Q} \mu_{\mathrm{a}, q} \phi_{q}(r), \\
& \mu_{\mathrm{s}}^{\prime}(r) \approx \mu_{\mathrm{s}}^{\prime h}(r)=\sum_{q=1}^{Q} \mu_{\mathrm{s}, q}^{\prime} \phi_{q}(r), \\
& \Phi(r) \approx \Phi^{h}(r)=\sum_{q=1}^{Q} \Phi_{q} \phi_{q}(r),
\end{aligned}
$$

where $\phi_{q}$ is the base function corresponding to grid node $r_{q}$, and $\mu_{\mathrm{a}, q}, \mu_{\mathrm{s}, q}^{\prime}$ and $\Phi_{q}$ are the discrete representations of $\mu_{\mathrm{a}}$, 
$\mu_{\mathrm{s}}^{\prime}$ and $\Phi$ respectively. The discretized absorbed energy density field (2) can then be approximated as

$$
H^{h}\left(r ; \mu_{\mathrm{a}}^{h}, \mu_{\mathrm{s}}^{\prime h}, s\right)=\left(\sum_{q=1}^{Q} \mu_{\mathrm{a}, q} \phi_{q}(r)\right)\left(\sum_{q=1}^{Q} \Phi_{q} \phi_{q}(r)\right),
$$

where $\Phi_{q}$ is the FEM solution of (1) with parameters $\mu_{\mathrm{a}}^{h}, \mu_{\mathrm{s}}^{\prime h}$ and $s$.

\section{B. Acoustic Model}

Propagation of sound, created by the instantaneous photoacoustic effect, in an infinite domain composed of homogeneous non-attenuating medium is described by the acoustic initial value problem [2], [5], [79]

$$
\left\{\begin{array}{l}
\frac{1}{c^{2}} \frac{\partial^{2}}{\partial t^{2}} p-\nabla^{2} p=0, \quad r \in \mathbb{R}^{n} \\
p(r, t=0)=p_{0}(r), \\
\frac{\partial}{\partial t} p(r, t=0)=0,
\end{array}\right.
$$

where $p$ is the acoustic pressure, $c$ is the sound speed, $t$ is the time, and $p_{0}$ is the initial pressure distribution created by the absorption of light pulse. The initial pressure distribution is given by

$$
p_{0}(r)= \begin{cases}\gamma H(r), & r \in \Omega \\ 0, & r \in \mathbb{R}^{n} \backslash \Omega\end{cases}
$$

where $\gamma$ is the Grüneisen parameter, and $H$ is the absorbed energy density as in (2). Throughout this work $\gamma$ is treated as a known constant, although in general, this is not the case.

In this work, the solution of (5) is approximated as a solution of the wave equation [80]

$$
\frac{1}{c^{2}} \frac{\partial^{2}}{\partial t^{2}} p-\nabla^{2} p=\frac{1}{c^{2}} p_{0}(r) \frac{\partial}{\partial t} g_{\tau}(t)
$$

where $g_{\tau}(t)$ is a (temporal) Gaussian distribution, with full width at half maximum $\tau$, approximating the formation of the initial pressure distribution $p_{0}$ into the acoustic medium. As $\tau$ approaches zero, (7) becomes a better approximation to $(5)$.

Solution of (7) can be computed as a spatial convolution [81]

$$
p(r, t)=p_{0}(r) * G_{\tau}(r, t)=\int_{\Omega} p_{0}\left(r^{\prime}\right) G_{\tau}\left(r-r^{\prime}, t\right) \mathrm{d} r^{\prime},
$$

where $G_{\tau}$ is the Green's function corresponding to (7), which can be expressed as

$$
G_{\tau}\left(r-r^{\prime}, t\right)=\frac{1}{c^{2}} \mathcal{F}^{-1}\left\{j \omega \hat{g}_{\tau}(\omega) \hat{\mathcal{G}}\left(\left\|r-r^{\prime}\right\|, \omega\right)\right\}(t),
$$

where $\mathcal{F}^{-1}$ is the (temporal) inverse Fourier transformation, $J$ is the imaginary unit, $\hat{g}_{\tau}$ is the Fourier transformation of $g_{\tau}$. $\hat{\mathcal{G}}$, in (9), is defined as

$$
\hat{\mathcal{G}}\left(\left\|r-r^{\prime}\right\|, \omega\right)= \begin{cases}J \frac{1}{4} H_{0}^{(1)}\left(\frac{\omega}{c}\left\|r-r^{\prime}\right\|\right), & n=2, \\ \frac{1}{4 \pi\left\|r-r^{\prime}\right\|} \exp \left(J \frac{\omega}{c}\left\|r-r^{\prime}\right\|\right), & n=3,\end{cases}
$$

which is the solution of $\nabla^{2} \hat{\mathcal{G}}+\frac{\omega^{2}}{c^{2}} \hat{\mathcal{G}}=-\delta(r)$ [81], $H_{0}^{1}$ is Hankel function of the first kind.
In this work, $p_{0}(r)$ in (7) is approximated using the same base functions as in the optical model as

$$
p_{0}(r) \approx p_{0}^{h}(r)=\sum_{q=1}^{Q} p_{0, q} \phi_{q}(r)
$$

Thus, (8) is approximated as

$$
p(r, t) \approx \sum_{q=1}^{Q} p_{0, q} \int_{\Omega} \phi_{q}\left(r^{\prime}\right) G_{\tau}\left(r-r^{\prime}, t\right) \mathrm{d} r^{\prime},
$$

with the spatial integral approximated using Gaussian quadratures. The Fourier transforms, in (9), are approximated by using discrete Fourier transformation corresponding to the temporal discretization. In discrete form, the solution (8) at a position $r$ can be written as

$$
\mathbf{p}(r)=\mathbf{w}(r) \mathbf{p}_{\mathbf{0}}
$$

where $\mathbf{p}(r)=\left(p\left(r, t_{1}\right), \ldots, p\left(r, t_{T}\right)\right)^{\top} \in \mathbb{R}^{T}$ is a vector with values of the acoustic pressure at time instances $t_{1}, \ldots, t_{T}$, and $\mathbf{w}(r) \in \mathbb{R}^{T \times Q}$ is a matrix describing the convolution integral in (8) as a linear operator, and $\mathbf{p}_{\mathbf{0}}=\left(p_{0,1}, \ldots, p_{0, Q}\right)^{\top} \in \mathbb{R}^{Q}$ is the discrete initial pressure distribution (11) as a vector.

\section{Photoacoustic Simulation Model}

In this work, the simulations are carried out in a photoacoustic setting composed of a light absorbing target surrounded by varying amount, as well as arrangement, of point size acoustic detectors. The target and the detectors are assumed to be immersed in an infinite homogeneous and nonattenuating acoustic medium, and the target is assumed to have the same acoustic parameters as the medium surrounding it. The mathematical model for this system is the combination of (1) and (5).

Defining the acoustic detectors as being located at positions $d_{k} \in \mathbb{R}^{n}$, with $k=1, \ldots, K$, the photoacoustic time series for illumination $s_{m}$, with $m=1, \ldots, M$, can be expressed as

$$
\mathbf{P}\left(\mu_{\mathrm{a}}^{h}, \mu_{\mathrm{s}}^{\prime h}, s_{m}\right)=\mathbf{W}_{\mathbf{0}}\left(\mu_{\mathrm{a}}^{h}, \mu_{\mathrm{s}}^{\prime h}, s_{m}\right),
$$

where $\mu_{\mathrm{a}}^{h}$ and $\mu_{\mathrm{s}}^{\prime h}$ are the optical parameters and

$$
\begin{aligned}
\mathbf{P}\left(\mu_{\mathrm{a}}^{h}, \mu_{\mathrm{s}}^{\prime h}, s_{m}\right) & =\left(\mathbf{p}\left(d_{1}\right)^{\top}, \ldots, \mathbf{p}\left(d_{K}\right)^{\top}\right)^{\top} \in \mathbb{R}^{T K}, \\
\mathbf{W} & =\left(\mathbf{w}\left(d_{1}\right)^{\top}, \ldots, \mathbf{w}\left(d_{K}\right)^{\top}\right)^{\top} \in \mathbb{R}^{T K \times Q},
\end{aligned}
$$

are as in (13) and

$$
\mathbf{p}_{\mathbf{0}}\left(\mu_{\mathrm{a}}^{h}, \mu_{\mathrm{s}}^{\prime h}, s_{m}\right)=\left(\gamma \mu_{\mathrm{a}, 1} \Phi_{m, 1}, \ldots, \gamma \mu_{\mathrm{a}, Q} \Phi_{m, Q}\right)^{\top} \in \mathbb{R}^{Q},
$$

is a vector representation of the discretization of (6). Note that in (16) $\mu_{\mathrm{a}, q} \Phi_{m, q}=H^{h}\left(r_{q} ; \mu_{\mathrm{a}}^{h}, \mu_{\mathrm{s}}^{\prime h}, s_{m}\right)$.

The photoacoustic simulation model (14) can be written, for illumination $s_{m}$, shortly as

$$
z_{m}=f_{m}(x),
$$

where $z_{m}=\mathbf{P}\left(\mu_{\mathrm{a}}^{h}, \mu_{\mathrm{s}}^{\prime h}, s_{m}\right) \in \mathbb{R}^{T K}, f_{m}(x)=$ $\mathbf{W p}_{\mathbf{0}}\left(\mu_{\mathrm{a}}^{h}, \mu_{\mathrm{s}}^{\prime h}, s_{m}\right) \quad: \quad \mathbb{R}^{2 Q} \rightarrow \mathbb{R}^{T K}$, and $x=$ $\left(\mu_{\mathrm{a}, 1}, \ldots, \mu_{\mathrm{a}, Q}, \mu_{\mathrm{s}, 1}^{\prime}, \ldots, \mu_{\mathrm{s}, Q}^{\prime}\right)^{\top} \in \mathbb{R}^{2 Q}$. For multiple illuminations, the forward model can be written as

$$
z=f(x),
$$


where $z=\left(z_{1}^{\top}, \ldots, z_{M}^{\top}\right)^{\top} \in \mathbb{R}^{T K M}$, and $f(x)=$ $\left(f_{1}(x)^{\top}, \ldots, f_{M}(x)^{\top}\right)^{\top}: \mathbb{R}^{2 Q} \rightarrow \mathbb{R}^{T K M}$.

\section{RECONSTRUCTION METHOD}

The inverse problem in QPAT, is to form estimates of the optical absorption and scattering based on the data. In this work, the inverse problem is approached in the Bayesian framework for ill-posed inverse problems.

\section{A. Bayesian Approach}

Solving an inverse problem of determining unknown parameter distribution $x \in \mathbb{R}^{2 Q}$ given noisy measurements, or data, $y \in \mathbb{R}^{T K M}$ requires definition of an observation model linking the parameters and the measurements. In this work, an observation model with additive error is used and it is written as

$$
y=f(x)+e,
$$

where $f(x): \mathbb{R}^{2 Q} \rightarrow \mathbb{R}^{T K M}$ is the (deterministic) forward model (18), and $e \in \mathbb{R}^{T K M}$ is a random variable denoting the additive error, or noise. The observation model (19) links the parameters of interest to the measurements.

Within the Bayesian approach [74]-[76], the forward model (19) is interpreted statistically by defining probability distributions for the unknown parameter $x$ and the noise $e$. The probability distribution of $x$ is called the prior distribution and it is intended to describe the existing (rough) knowledge of the unknown $x$, while the noise statistics essentially characterises the measurement setup and modelling errors. The prior and the noise probability density function are marked with $\pi_{x}(x)$ and $\pi_{e}(e)$ respectively. Assuming that $x$ and $e$ are uncorrelated in the additive noise model (19), the Bayesian approach results in posterior distribution $\pi(x \mid y)$ for the unknown $x$ conditioned by the measurements $y$, and it is given by

$$
\pi(x \mid y) \propto \pi_{x}(x) \pi_{e}(y-f(x)) .
$$

In principle, the posterior distributions of the unknown parameters can be estimated using Markov chain Monte Carlo (MCMC) methods. However, these methods can be computationally prohibitively too expensive in large dimensional tomographic inverse problems. Therefore, point estimates such as the maximum a posteriori (MAP) estimate are computed. In this work, the distributions $\pi_{x}$ and $\pi_{e}$ are modelled as Gaussian distributions, and their parameters are denoted with $x \sim \mathcal{N}\left(\eta_{x}, \Gamma_{x}\right)$ and $e \sim \mathcal{N}\left(\eta_{e}, \Gamma_{e}\right)$, where $\eta_{x}$ and $\Gamma_{x}$ are the mean and the covariance of the prior information discussed in Section III-B, and $\eta_{e}$ and $\Gamma_{e}$ are the mean and the covariance characterizing the noise and uncertainties of the measurement setup. Values for $\eta_{e}$ and $\Gamma_{e}$, characterizing the additive error in (19) are defined in Section IV. With the Gaussian choice for the distributions, the negative logarithm of the posterior distribution (20) becomes

$$
u(x)=\frac{1}{2}\left\|L_{e}\left(y-f(x)-\eta_{e}\right)\right\|^{2}+\frac{1}{2}\left\|L_{x}\left(x-\eta_{x}\right)\right\|^{2},
$$

where $L_{e}$ and $L_{x}$ are the Cholesky decompositions of the inverse covariance matrices, such that $L_{e}^{\top} L_{e}=\Gamma_{e}^{-1}$ and
$L_{x}^{\top} L_{x}=\Gamma_{x}^{-1}$. The point estimate of $x$ used in this paper is the MAP estimate

$$
x_{\mathrm{MAP}}=\arg \min _{x} u(x),
$$

which is obtained by using a Gauss-Newton algorithm [82]. The Gauss-Newton proceeds by starting from some initial value $x=x_{1}$ and using iteration

$$
x_{l+1}=x_{l}+\alpha_{l} \Delta_{l}, \quad l \geq 1
$$

where $\alpha_{l}$ is obtained by using a line search algorithm to find minimum of $u\left(x_{l}+\alpha_{l} \Delta_{l}\right) . \Delta_{l}$ in (23) is the Gauss-Newton iteration direction at step $l$ computed by solving

$$
\begin{aligned}
\left(J_{f\left(x_{l}\right)}^{\top} \Gamma_{e}^{-1} J_{f\left(x_{l}\right)}+\Gamma_{x}^{-1}\right) \Delta_{l}= & J_{f\left(x_{l}\right)}^{\top} \Gamma_{e}^{-1}\left(y-f\left(x_{l}\right)-\eta_{e}\right) \\
& -\Gamma_{x}^{-1}\left(x-\eta_{x}\right),
\end{aligned}
$$

where $J_{f\left(x_{l}\right)}$ is the Jacobian matrix of $f(x)$ at $x=x_{l}$. An approximation $x_{\text {MAP }} \approx x_{l}$ is then obtained with sufficiently large $l$.

One of the main assets of employing the Bayesian approach is that, in addition to point estimates, one can also compute (approximations for) the posterior error estimates of the unknown parameters of interest. In Bayesian analysis, the credibility intervals (counterparts of confidence intervals in frequentist statistics) would be the standard choice for error estimates. The computation of credibility intervals would, however, call for Markov chain Monte Carlo methods which, in our case, are not computationally feasible approaches. Instead, in this paper, we compute approximations for the credibility intervals that are based on local Gaussian approximation of the posterior distribution at the MAP estimate. The computation of these approximations can be derived as follows. The forward model $x \mapsto y=f(x)$ is approximated by the first order Taylor series at $x_{\text {MAP }}$

$$
f(x) \approx f\left(x_{\mathrm{MAP}}\right)+J_{f\left(x_{\mathrm{MAP}}\right)}\left(x-x_{\mathrm{MAP}}\right)
$$

where $J_{f\left(x_{\mathrm{MAP}}\right)}$ is the Jacobian matrix of $f(x)$ evaluated at $x=x_{\text {MAP }}$, which is obtained using the Gauss-Newton algorithm. Then, the Taylor series approximation is substituted into the observation model (19). By forming the mean and covariance matrix of the combined distribution of $(x, y)$, and then using Schur complements to obtain the conditional distribution of $x \mid y$ results in a Gaussian approximation for the posterior distribution, that is, $x \mid y \sim \mathcal{N}(\eta, \Gamma)$, where the (approximate) posterior mean is $\eta=x_{\mathrm{MAP}}$ and the posterior covariance is

$$
\Gamma=\left(J_{f\left(x_{\mathrm{MAP}}\right)}^{\top} \Gamma_{e}^{-1} J_{f\left(x_{\mathrm{MAP}}\right)}+\Gamma_{x}^{-1}\right)^{-1} .
$$

For full derivation, see [74], [75]. For true Gaussian distributions, $99.7 \%$ of the probability mass of each element $x_{i}$ of $x$ would lie in the interval $\eta_{i} \pm 3 \sigma_{i}$ where $\sigma_{i}=\sqrt{\Gamma(i, i)}$. In this paper, we refer to these intervals as posterior error estimates. Although these intervals do not exactly correspond to $99.7 \%$ of the respective probability mass, such posterior error intervals can be described as safe ones. The validity 
of the approximation is evaluated in the Appendix. Thus, we define the posterior error intervals at points $r_{q}$ as

$$
\begin{aligned}
& C_{\mu_{\mathrm{a}}}\left(r_{q}\right)=\left[\mu_{\mathrm{a}, q}^{\mathrm{MAP}}-3 \sigma_{\mathrm{a}, q}, \mu_{\mathrm{a}, q}^{\mathrm{MAP}}+3 \sigma_{\mathrm{a}, q}\right], \\
& C_{\mu_{\mathrm{s}}^{\prime}}\left(r_{q}\right)=\left[\mu_{\mathrm{s}, q}^{\prime}{ }^{\prime \mathrm{MAP}}-3 \sigma_{\mathrm{s}, q}, \mu_{\mathrm{s}, q}^{\prime \mathrm{MAP}}+3 \sigma_{\mathrm{s}, q}\right],
\end{aligned}
$$

where $\sigma_{\mathrm{a}, q}$ and $\sigma_{\mathrm{s}, q}$ are the square roots of the respective diagonal elements of the covariance matrix $\Gamma$.

\section{B. Prior Model}

In this work, the prior model for the unknown parameters $\mu_{\mathrm{a}}$ and $\mu_{\mathrm{s}}^{\prime}$ was chosen to be based on the Ornstein-Uhlenbeck process [45], [63], [83]. The prior is a Gaussian distribution with the covariance matrix defined as being proportional to matrix $\Xi$ which has its elements defined as

$$
\Xi_{i j}=\exp \left(-\left\|r_{i}-r_{j}\right\| / \xi\right)
$$

where $i$ and $j$ denote the row and column indices of the matrix, $r_{i}$ and $r_{j}$ denote the grid node coordinates, and $\xi$ is the characteristic length scale of the prior describing the spatial distance that the parameter is expected to have (significant) spatial correlation for. The random field described by the covariance (28) is independent of the absolute position, with the spatial correlation between any two points $r_{i}$ and $r_{j}$ being only dependent on the distance between the points, and the characteristic length scale. The characteristic length scale is typically chosen to be descriptive of the expected size of inclusions in the target of interest.

In this work, the mean of the priors for $\mu_{\mathrm{a}}$ and $\mu_{\mathrm{s}}^{\prime}$ were chosen as

$$
\begin{aligned}
& \eta_{\mu_{\mathrm{a}}}=\frac{1}{2}\left(\max \mu_{\mathrm{a}}+\min \mu_{\mathrm{a}}\right), \\
& \eta_{\mu_{\mathrm{s}}^{\prime}}=\frac{1}{2}\left(\max \mu_{\mathrm{s}}^{\prime}+\min \mu_{\mathrm{s}}^{\prime}\right),
\end{aligned}
$$

and the covariances of the priors were chosen as

$$
\begin{gathered}
\Gamma_{\mu_{\mathrm{a}}}=\frac{1}{4}\left(\max \mu_{\mathrm{a}}-\min \mu_{\mathrm{a}}\right)^{2} \Xi, \\
\Gamma_{\mu_{\mathrm{s}}^{\prime}}=\frac{1}{4}\left(\max \mu_{\mathrm{s}}^{\prime}-\min \mu_{\mathrm{s}}^{\prime}\right)^{2} \Xi,
\end{gathered}
$$

where $\min \mu_{\mathrm{a}}, \max \mu_{\mathrm{a}}, \min \mu_{\mathrm{s}}^{\prime}$, and $\max \mu_{\mathrm{s}}^{\prime}$ denote the minimum and maximum values that $\mu_{\mathrm{a}}$ and $\mu_{\mathrm{s}}^{\prime}$ are expected to vary between, with the minimum and maximum being one standard deviation away from their midpoint. The combined prior parameters were then

$$
\eta_{x}=\left(\begin{array}{c}
\eta_{\mu_{\mathrm{a}}} \\
\eta_{\mu_{\mathrm{s}}^{\prime}}
\end{array}\right), \Gamma_{x}=\left(\begin{array}{cc}
\Gamma_{\mu_{\mathrm{a}}} & 0 \\
0 & \Gamma_{\mu_{\mathrm{s}}^{\prime}}
\end{array}\right) .
$$

These choices correspond to prior assumption that $\mu_{\mathrm{a}}$ and $\mu_{\mathrm{s}}^{\prime}$ are mutually uncorrelated. In biological tissues, this assumption can be inaccurate in the sense that close to tissue boundaries it is reasonable to expect that both of the optical properties could change. This would imply some correlation between the optical parameters, however, the uncorrelation assumption of the optical properties promotes more generic solutions to the inverse problem and is thus used in this work.
TABLE I

THE ILLUMINATION FUnCTIONS USED

\begin{aligned} & \hline Illuminating sides Illumination functions \\ & \hline \hline \multirow{2}{*}{ Four sided }$s_{\mathrm{LR}}^{2 \mathrm{D}}(r)=s\left(r ; \Omega_{\mathrm{L}} \cup \Omega_{\mathrm{R}}\right) \\ & s_{\mathrm{BT}}^{2 \mathrm{D}}(r)=s\left(r ; \Omega_{\mathrm{B}} \cup \Omega_{\mathrm{T}}\right) \\ &$\hline \multirow{2}{*}{ Three sided }$s_{\mathrm{LB}}^{2 \mathrm{D}}(r)=s\left(r ; \Omega_{\mathrm{L}} \cup \Omega_{\mathrm{B}}\right) \\ & s_{\mathrm{T}}^{2 \mathrm{D}}(r)=s\left(r ; \Omega_{\mathrm{T}}\right) \\ &$\hline \multirow{2}{*}{ Two sided }$s_{\mathrm{L}}^{2 \mathrm{D}}(r)=s\left(r ; \Omega_{\mathrm{L}}\right) \\ & s_{\mathrm{B}}^{2 \mathrm{D}}(r)=s\left(r ; \Omega_{\mathrm{B}}\right) \\ &$\hline \multirow{2}{*}{ Six sided }$s_{\mathrm{LBD}}^{3 \mathrm{D}}(r)=s\left(r ; \Omega_{\mathrm{L}} \cup \Omega_{\mathrm{B}} \cup \Omega_{\mathrm{D}}\right) \\ & s_{\mathrm{RTV}}^{3 \mathrm{D}}(r)=s\left(r ; \Omega_{\mathrm{R}} \cup \Omega_{\mathrm{T}} \cup \Omega_{\mathrm{V}}\right) \\ &$\hline\end{aligned}

\section{Simulation Studies}

The approach was studied using $2 \mathrm{D}$ and 3D simulations. The inverse problem of estimating $\mu_{\mathrm{a}}$ and $\mu_{\mathrm{s}}^{\prime}$ using the models described in Section II and the reconstruction method described in Section III was investigated. In 2D, the acoustic sensor configuration, optical illuminations, and the noise level were varied. In 3D, a limited view case with acoustic measurement performed only from one side of the target was investigated.

For the inverse problems, the MAP estimates (22) were computed using the Gauss-Newton algorithm (23). GaussNewton algorithm started from initial value of $x_{1}=\eta_{x}$. Accuracy of the estimates were computed using relative errors

$$
\begin{aligned}
& E_{\mu_{\mathrm{a}}}=100 \% \cdot \frac{\left\|\mu_{\mathrm{a}}^{\mathrm{MAP}}-\mu_{\mathrm{a}}^{\mathrm{TRUE}}\right\|}{\left\|\mu_{\mathrm{a}}^{\mathrm{TRUE}}\right\|}, \\
& E_{\mu_{\mathrm{s}}^{\prime}}=100 \% \cdot \frac{\left\|\mu_{\mathrm{s}}^{\prime \mathrm{MAP}}-\mu_{\mathrm{s}}^{\prime \mathrm{TRUE}}\right\|}{\left\|\mu_{\mathrm{s}}^{\prime \mathrm{TRUE}}\right\|},
\end{aligned}
$$

where $\mu_{\mathrm{a}}^{\mathrm{TRUE}}$ and $\mu_{\mathrm{s}}^{\prime \mathrm{TRUE}}$ are the true parameters used to compute the simulated measurement data and $\mu_{\mathrm{a}}^{\mathrm{MAP}}$ and $\mu_{\mathrm{s}}^{\prime \mathrm{MAP}}$ are their respective MAP estimates, obtained with (22).

\section{A. $2 D$ Simulations}

The 2D simulations were performed in square domain $\Omega=[-5 \mathrm{~mm}, 5 \mathrm{~mm}] \times[-5 \mathrm{~mm}, 5 \mathrm{~mm}]$. Two different illuminations $(M=2)$ were used to generate the simulated measurement data. The illuminations were formed by functions

$$
s(r ; \Lambda)=\left\{\begin{array}{ll}
1 \frac{\mathrm{J}}{\mathrm{mm}^{2}}, & r \in \Lambda \\
0 \frac{\mathrm{J}}{\mathrm{mm}^{2}}, & r \notin \Lambda
\end{array},\right.
$$

with $\Lambda$ being combination of $\Omega_{\mathrm{L}}, \Omega_{\mathrm{R}}, \Omega_{\mathrm{B}}, \Omega_{\mathrm{T}}$ corresponding to left, right, bottom and top edges of the square domain respectively. The illumination functions are listed in Table I.

For the acoustic sensor locations, six different configurations were used: four densely positioned detector configurations and two sparsely positioned detector configurations. The densely positioned detector configurations were: four sided detection geometry with sensors on $\Omega_{\mathrm{L}}, \Omega_{\mathrm{R}}, \Omega_{\mathrm{B}}, \Omega_{\mathrm{T}}$ (number of sensors is $K=128$ ); three sided detection geometry with sensors on $\Omega_{\mathrm{L}}, \Omega_{\mathrm{B}}, \Omega_{\mathrm{T}}(K=96)$; two sided detection 


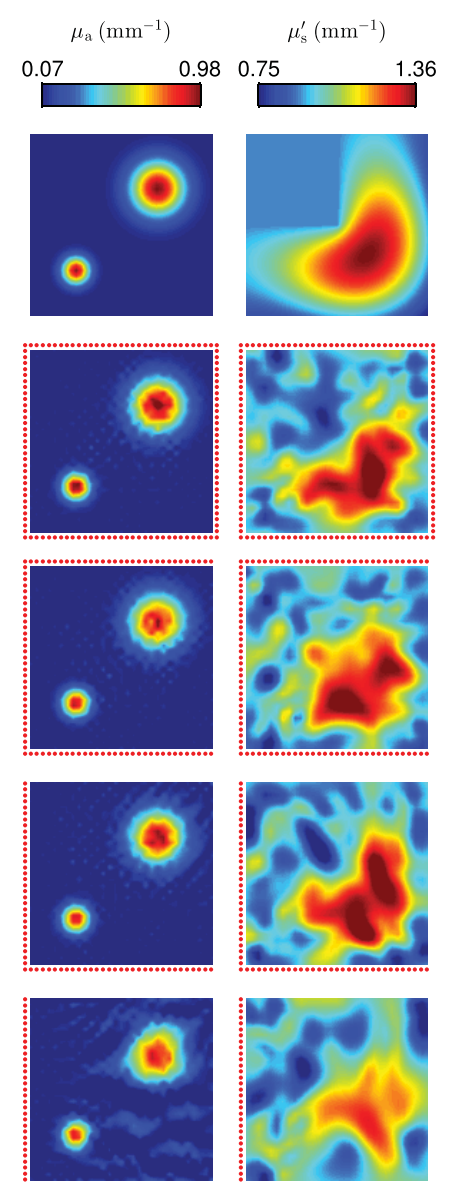

Fig. 1. MAP estimates for limited view acoustic detection setups. Shown are the true optical parameters used to simulate the data (top row), followed by MAP estimates with acoustic measurement obtained on four sides (second row), three sides (third row), two sides (fourth row), and one side (bottom row). The locations of the acoustic sensors are indicated with the red dots. Four illuminating sides were used $\left(s_{\mathrm{LR}}^{2 \mathrm{D}}\right.$ and $\left.s_{\mathrm{BT}}^{2 \mathrm{D}}\right)$. The MAP estimates were computed based on data corrupted by $1 \%$ noise.

geometry with sensors on $\Omega_{\mathrm{L}}, \Omega_{\mathrm{B}}(K=63)$; one sided detection geometry with sensors on $\Omega_{\mathrm{L}}(K=31)$. The sparsely positioned detector configurations were: sensors on a circle with a radius of $7.8 \mathrm{~mm}(K=31)$; and sensors on a semicircle with a radius of $7.8 \mathrm{~mm}(K=15)$. The sensor configurations are shown in Figures 1 and 2 with red dots.

The data was simulated in a grid composed of $Q=1369$ grid nodes formed by square elements with side length of $277.8 \mu \mathrm{m}$. The distribution of $\mu_{\mathrm{a}}$ and $\mu_{\mathrm{s}}^{\prime}$ parameters are shown in Figure 1 on the top row. The parameter values were chosen to be within biologically relevant variation ranges of fat and blood [84]. For the sound speed, value $c=1500 \mathrm{~m} / \mathrm{s}$ was used, which is similar to the speed of sound in water and soft tissues. The temporal pressure waveforms were sampled at $10 \mathrm{MHz}$ and discretized into $T=189$ temporal points at each of the acoustic sensor location. This corresponds to a recorded temporal pressure time series duration of $18.9 \mu$ s and an acoustic propagation distance of $28.4 \mathrm{~mm}$. The temporally Gaussian light pulse $g_{\tau}$ had full width at half maximum of $\tau=333 \mathrm{~ns}$. Normally distributed noise, with zero mean and standard deviation proportional to the peak-to-peak pressure

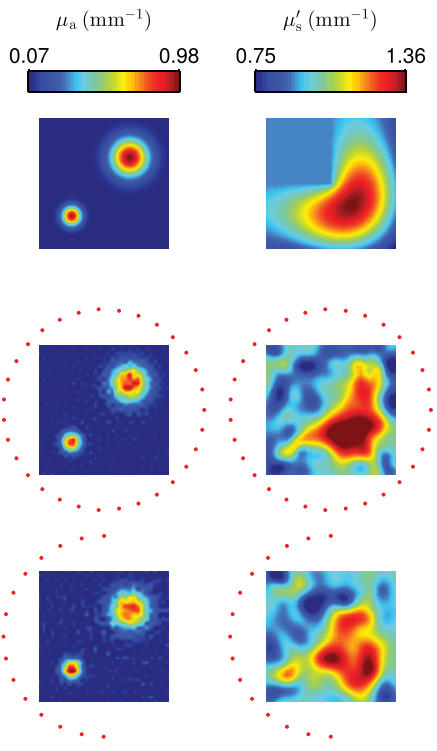

Fig. 2. MAP estimates for sparsely located acoustic detection setups. Shown are the true optical parameters used to simulate the data (top row), and MAP estimate when using circularly (middle row) and semicircularly (bottom row) arranged set of acoustic sensors. The locations of the acoustic sensors are indicated with the red dots. Four illuminating sides were used $\left(s_{\mathrm{LR}}^{2 \mathrm{D}}\right.$ and $\left.s_{\mathrm{BT}}^{2 \mathrm{D}}\right)$. The MAP estimates were computed based on data corrupted by $1 \%$ noise.

amplitude of the detected acoustic time series, was added to the simulated measurement data. This was done for the data obtained with the two different illuminations separately. The standard deviation was thus defined as

$$
\sigma_{m}=\epsilon\left(\max \mathbf{P}\left(\mu_{\mathrm{a}}^{h}, \mu_{\mathrm{s}}^{\prime h}, s_{m}\right)-\min \mathbf{P}\left(\mu_{\mathrm{a}}^{h}, \mu_{\mathrm{s}}^{\prime h}, s_{m}\right)\right),
$$

where $\epsilon$ is the noise level and $m \in\{1,2\}$. Values of $\epsilon=0.05$, 0.01 and 0.001 were used corresponding to $5 \%, 1 \%$ and $0.1 \%$ noise levels, or 13, 20 and $30 \mathrm{~dB}$ signal-to-noise ratios (SNR) respectively.

In the inverse problem, a grid composed of $Q=961$ grid nodes formed by square elements with side length of $333.3 \mu \mathrm{m}$ was used. For the noise statistics, the following values were used:

$$
\eta_{e}=0, \quad \Gamma_{e}=\left(\begin{array}{cc}
\sigma_{1}^{2} I & 0 \\
0, & \sigma_{2}^{2} I
\end{array}\right),
$$

where $\sigma_{1}$ and $\sigma_{2}$ are the standard deviation of the added noise corresponding to the two illuminations used, and $I \in \mathbb{R}^{Q \times Q}$ is the identity matrix. These values correspond to accurately knowing the statistics of the noise. The prior was assembled according to (31), with the characteristic length scale $\xi=1 \mathrm{~mm}$. For the expected range of variation, the true minimum and maximum of the estimated parameters were used resembling a good prior knowledge.

\section{B. $3 D$ Simulations}

The 3D simulations were performed in a cube domain $\Omega=[-5 \mathrm{~mm}, 5 \mathrm{~mm}] \times[-5 \mathrm{~mm}, 5 \mathrm{~mm}] \times[-5 \mathrm{~mm}, 5 \mathrm{~mm}]$. Two different illumination functions $(M=2), s_{\mathrm{LBD}}^{3 \mathrm{D}}$ and $s_{\mathrm{RTV}}^{3 \mathrm{D}}$, were used to generate the simulated measurement data which are shown in Table I with $\Omega_{\mathrm{L}}, \Omega_{\mathrm{R}}, \Omega_{\mathrm{B}}, \Omega_{\mathrm{T}}, \Omega_{\mathrm{D}}, \Omega_{\mathrm{V}}$ corresponding to left, right, bottom, top, dorsal (rear) and 
ventral (front) faces of the cube. $K=1089$ acoustic sensors were located on $\Omega_{\mathrm{L}}$ face of the cube set in square $33 \times 33$ sensor array.

The 3D simulation domain was discretized into cube elements with side length $277.8 \mu \mathrm{m}$. This resulted in a simulation grid composed of $Q=50653$ grid nodes, which was used to compute the simulated measurements. The temporal data was sampled at $10 \mathrm{MHz}$ and discretized into $T=128$ temporal points, corresponding to a recorded temporal pressure time series duration of $12.7 \mu \mathrm{s}$ and an acoustic propagation distance of $19.1 \mathrm{~mm}$. The same full width at half maximum, as in 2D, of $\tau=333 \mathrm{~ns}$ was used for $g_{\tau}$. The parameters used for $\mu_{\mathrm{a}}$ and $\mu_{\mathrm{s}}^{\prime}$ to compute the data are shown in Figure 6. Noise was added in the same fashion as in 2D with the noise level $\epsilon=0.001$ corresponding to $0.1 \%$ noise level.

In the inverse problem, the simulation grid was composed of $Q=29791$ grid nodes. The statistics of the noise mean and covariance matrix were chosen as in (35), and the prior was assembled according to (31), with the characteristic length scale $\xi=1 \mathrm{~mm}$. For the expected range of variation, the true minimum and maximum of the estimated parameters were used.

\section{RESULTS}

2D MAP estimates of $\mu_{\mathrm{a}}$ and $\mu_{\mathrm{s}}^{\prime}$ are shown in Figure 1 for the data with noise level at $1 \%$. Illuminations $s_{\mathrm{LR}}^{2 \mathrm{D}}$ and $s_{\mathrm{BT}}^{2 \mathrm{D}}$ were used. Results of four simulations where the acoustic sensors were located on the sides of the square domain $\Omega$ are shown. Visually the estimates of $\mu_{\mathrm{a}}$ look comparable to each other even in the case when the acoustic sensors have located on only one side of the imaged target. In the one sided sensor configuration, however, some artefacts in the estimate are evident and visually look as if streaming away from the sensors. Larger differences between the estimates of $\mu_{\mathrm{s}}^{\prime}$ obtained with different acoustic sensor configurations are evident, but even in the case of one sided acoustic sensor configuration the estimate resembles the true $\mu_{\mathrm{s}}^{\prime}$.

2D MAP estimates of $\mu_{\mathrm{a}}$ and $\mu_{\mathrm{s}}^{\prime}$ are shown in Figure 2 for sensor configuration where the sensors were located on a circle or semicircle. The noise level was $1 \%$ and illuminations $s_{\mathrm{LR}}^{2 \mathrm{D}}$ and $s_{\mathrm{BT}}^{2 \mathrm{D}}$ were used. In the case of the circle, the number of acoustic sensors was equal to that used in the one sided configuration. Visually the estimates of $\mu_{\mathrm{a}}$ and $\mu_{\mathrm{s}}^{\prime}$ are closer to the true parameters and contain less artefacts in comparison to one sided acoustic configuration. The estimate of $\mu_{\mathrm{s}}^{\prime}$ is closer in amplitude to the true value as well. In the case of semicircle, degradation of the visual quality of the estimate is evident in comparison to full circle configuration. However, although the semicircle configuration has less sensors than the one sided configuration in Figure 1, the estimate is better in visual quality.

Figure 3 shows 2D MAP estimates of $\mu_{\mathrm{a}}$ and $\mu_{\mathrm{s}}^{\prime}$ for noise levels $5 \%, 1 \%$ and $0.1 \%$ for two sided acoustic sensor configuration. Illuminations $s_{\mathrm{LR}}^{2 \mathrm{D}}$ and $s_{\mathrm{BT}}^{2 \mathrm{D}}$ were used. As it can be seen, at high noise levels, the estimates of $\mu_{\mathrm{s}}^{\prime}$ are smooth and blurry, whereas at the low noise level, the estimate becomes sharper. The effect suggests, that at high noise levels,

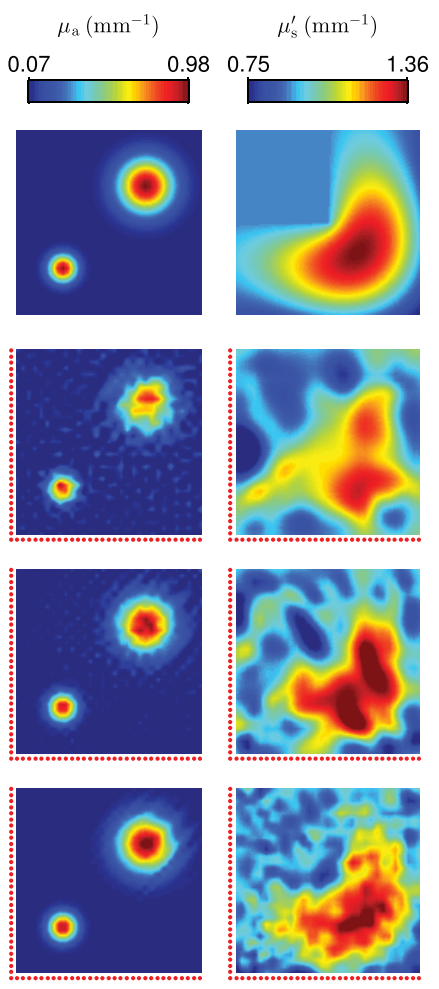

Fig. 3. MAP estimates for varying noise levels. Shown are the true optical parameters used to simulate the data (top row), followed by MAP estimates with the data being corrupted by $5 \%$ (second row), $1 \%$ (third row), and $0.1 \%$ (fourth row) noise. The locations of the acoustic sensors are indicated with the red dots. Four illuminating sides were used $\left(s_{\mathrm{LR}}^{2 \mathrm{D}}\right.$ and $\left.s_{\mathrm{BT}}^{2 \mathrm{D}}\right)$.

information about the high spatial frequency components of $\mu_{\mathrm{s}}^{\prime}$ is lost. Estimates of $\mu_{\mathrm{a}}$, on the other hand, appear to become polluted by spatially high frequency noise when the noise level is high. Figure 4 shows the posterior error intervals of the MAP estimates of Figure 3 on a diagonal of the domain $\Omega$, computed with (27). As the noise level decreases, the estimates become more accurate, the posterior error intervals decrease and, in particular, the point estimates are consistent with the error estimates. For the absorption coefficients, the posterior error intervals also reflect the intuitive hypothesis that the error intervals should be larger at points which are further away from the measurement locations. Also, as expected, the error intervals for the scattering coefficient are larger than for the absorption coefficient, and they decrease slower with decreasing noise level. Regarding the width of the error intervals, we remind that we employ $\pm 3 \sigma$ intervals which are expected to contain almost $100 \%$ of the probability mass.

Figure 5 shows 2D MAP estimates of $\mu_{\mathrm{a}}$ and $\mu_{\mathrm{s}}^{\prime}$ when number of illuminating sides has been varied. Estimates were computed with four side $\left(s_{\mathrm{LR}}^{2 \mathrm{D}}\right.$ and $\left.s_{\mathrm{BT}}^{2 \mathrm{D}}\right)$, three side $\left(s_{\mathrm{LB}}^{2 \mathrm{D}}\right.$ and $\left.s_{\mathrm{T}}^{2 \mathrm{D}}\right)$, and two side $\left(s_{\mathrm{L}}^{2 \mathrm{D}}\right.$ and $\left.s_{\mathrm{B}}^{2 \mathrm{D}}\right)$ illuminations. Noise level was $0.1 \%$. As the total illumination surface is reduced from four sided illumination to two sided illumination, the information in the areas far from the illuminating sides becomes poorer. This is reflected in estimates of $\mu_{\mathrm{a}}$ as becoming more distorted in those areas, as evident in the top right 

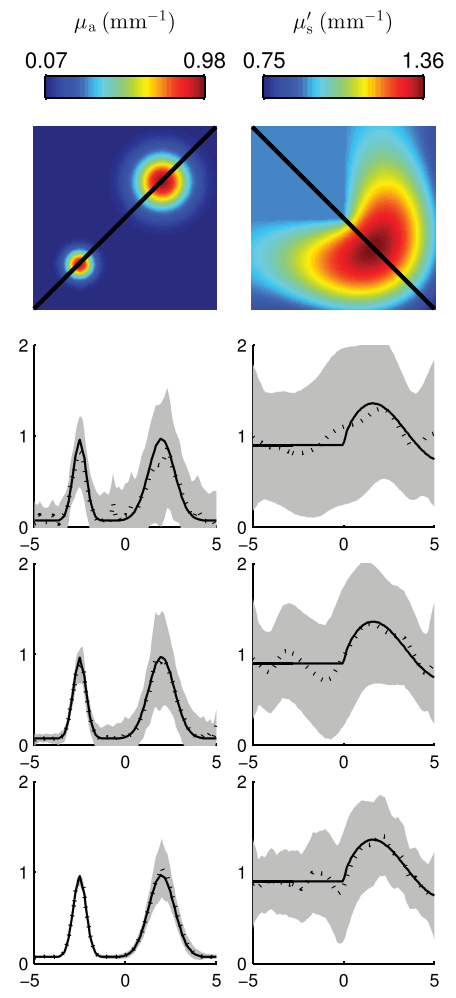

Fig. 4. Posterior error intervals for varying noise levels. Shown are the true optical parameters used to simulate the data (top row), followed by the approximate $\pm 3 \sigma$ posterior error intervals corresponding to $5 \%$ (second row), $1 \%$ (third row), and $0.1 \%$ noise levels. The points at which the error intervals are shown are on diagonal cross sections across the domain and are indicated as black lines in the top row images. The plots show the true parameters (solid line), the MAP estimates (dotted line), and the error intervals (grey areas). Four illuminating sides were used $\left(s_{\mathrm{LR}}^{2 \mathrm{D}}\right.$ and $\left.s_{\mathrm{BT}}^{2 \mathrm{D}}\right)$.

corner of the estimate produced by using illumination pair $s_{\mathrm{L}}^{2 \mathrm{D}}$ and $s_{\mathrm{B}}^{2 \mathrm{D}}$. Estimate of $\mu_{\mathrm{s}}^{\prime}$ becomes overall worse everywhere as the total illuminating surface is reduced, with no clearly visible spatial location where the estimate is worst.

3D MAP estimates of $\mu_{\mathrm{a}}$ and $\mu_{\mathrm{s}}^{\prime}$ are shown in Figure 6. Illuminations $s_{\mathrm{LBD}}^{3 \mathrm{D}}$ and $s_{\mathrm{RTV}}^{3 \mathrm{D}}$ were used. The figure depicts situation where the acoustic sensors are located on one side of the imaged target. The estimates of both parameters work reasonably well, although they both suffer from imaging artefacts caused by the limited view. The inclusions (volumes of higher absorption or scattering in comparison to the ambient value) are visible in both absorption and scattering estimates.

Table II shows the relative errors of the MAP estimates shown in Figures 1 and 2 for the six simulated 2D acoustic sensor geometries and one 3D simulation shown in Figure 6. For the 2D simulations the relative errors are shown for noise levels of $5 \%, 1 \%$ and $0.1 \%$. Illuminations $s_{\mathrm{LR}}^{2 \mathrm{D}}$ and $s_{\mathrm{BT}}^{2 \mathrm{D}}$ were used in $2 \mathrm{D}$ and $s_{\mathrm{LBD}}^{3 \mathrm{D}}$ and $s_{\mathrm{RTV}}^{3 \mathrm{D}}$ in $3 \mathrm{D}$. Based on the relative errors it is evident that the quality of the estimates of $\mu_{\mathrm{a}}$ and $\mu_{\mathrm{s}}^{\prime}$ improves when the noise level is reduced, or when the sensors are located in multiple directions of target. On the smallest error level of $0.1 \%$, the circular and semicircular detector geometries resulted in lower relative errors of the estimates than in the one sided detector geometry.

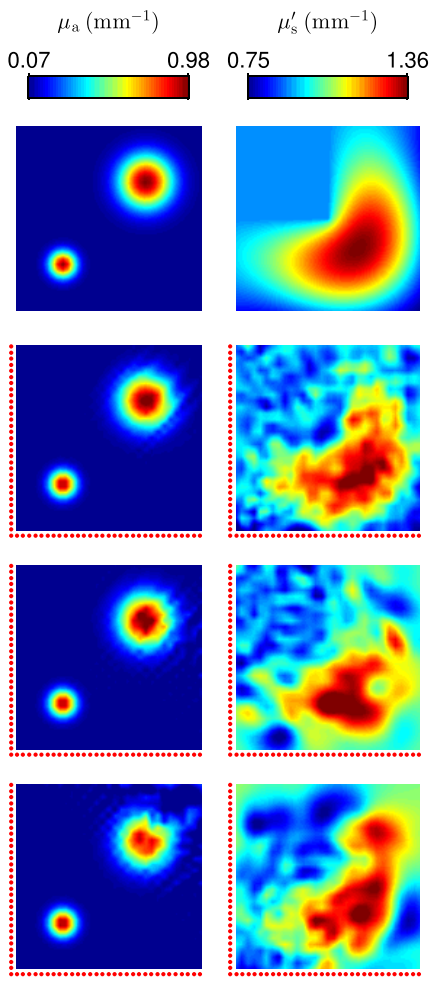

Fig. 5. MAP estimates for varying illuminations. Shown are the true optical parameters used to simulate the data (top row), followed by MAP estimates with four ( $s_{\mathrm{LR}}^{2 \mathrm{D}}$ and $s_{\mathrm{BT}}^{2 \mathrm{D}}$, second row), three $\left(s_{\mathrm{LB}}^{2 \mathrm{D}}\right.$ and $s_{\mathrm{T}}^{2 \mathrm{D}}$, third row), and two illuminating sides $\left(s_{\mathrm{L}}^{2 \mathrm{D}}\right.$ and $s_{\mathrm{B}}^{2 \mathrm{D}}$, fourth row). The locations of the acoustic sensors are indicated with the red dots. The MAP estimates were computed based on data corrupted by $0.1 \%$ noise.

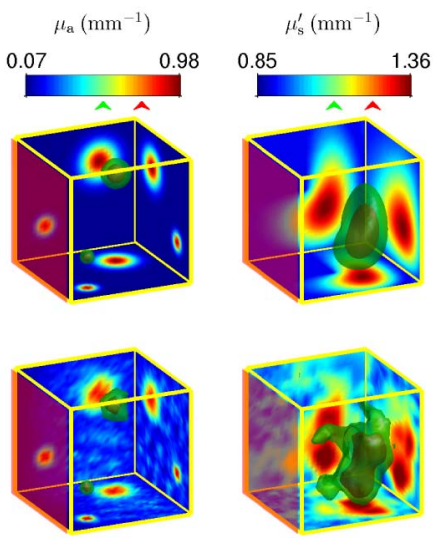

Fig. 6. MAP estimates in 3D. Shown are the true optical parameters used to simulate the data (top row), and the MAP estimates (bottom row). The three back faces of the cubes show the maximum value projection of the optical parameters along the perpendicular axis. The red and green contour surfaces denote the areas where the parameters have $75 \%$ and $50 \%$ values of the parameter range. These values are pointed out by the red and green arrows, respectively, beneath the colorbars. The red plane denotes the location of the acoustic sensors. Six illuminating sides were used $\left(s_{\mathrm{LBD}}^{3 \mathrm{D}}\right.$ and $\left.s_{\mathrm{RTV}}^{3 \mathrm{D}}\right)$. The MAP estimates were computed based on data corrupted by $0.1 \%$ noise.

Relative errors of $\mu_{\mathrm{a}}$ and $\mu_{\mathrm{s}}^{\prime}$ when the illuminating functions have been varied are shown in Table III for estimates shown in Figure 5. Based on the relative errors, it is evident that the estimates become worse when the total illuminating area is reduced or when the noise level is increased. 
TABLE II

RELATIVE ERRors $E_{\mu_{\mathrm{a}}}$ AND $E_{\mu_{\mathrm{s}}^{\prime}}$ FOR THE MAP ESTIMATES OF $\mu_{\mathrm{a}}$ AND $\mu_{\mathrm{s}}^{\prime}$ OBTAINED IN 2D FOR THE SiX DifFEREnT ACOUSTIC SENSOR CONFIGURATIONS AND FOR THE SIMULATION STUDY IN 3D. IN 2D, ILLUMINATIONS $s_{\mathrm{LR}}^{2 \mathrm{D}}$ AND $s_{\mathrm{BT}}^{2 \mathrm{D}}$ WERE USED, WHEREAS IN 3D $s_{\mathrm{LBD}}^{3 \mathrm{D}}$ AND $s_{\mathrm{RTV}}^{3 \mathrm{D}}$ WERE USED

\begin{tabular}{rcccccc}
\hline & \multicolumn{3}{c}{$E_{\mu_{\mathrm{a}}}(\%)$} & \multicolumn{3}{c}{$E_{\mu_{\mathrm{s}}^{\prime}}(\%)$} \\
Noise levels $\epsilon$ & $5 \%$ & $1 \%$ & $0.1 \%$ & $5 \%$ & $1 \%$ & $0.1 \%$ \\
\hline \hline 2D Four sided & 17.0 & 6.4 & 2.0 & 8.5 & 6.2 & 4.3 \\
2D Three sided & 18.0 & 7.7 & 1.7 & 11.4 & 8.0 & 4.4 \\
2D Two sided & 22.3 & 7.9 & 4.1 & 9.3 & 7.2 & 4.8 \\
2D One sided & 26.7 & 22.2 & 17.0 & 12.5 & 9.9 & 5.4 \\
2D Circle & 27.5 & 12.4 & 2.9 & 12.3 & 7.4 & 4.9 \\
2D Semicircle & 33.3 & 15.8 & 8.1 & 14.2 & 8.3 & 4.7 \\
\hline 3D & - & - & 55.5 & - & - & 6.9 \\
\hline
\end{tabular}

TABLE III

RELATIVE ERRORS $E_{\mu_{\mathrm{a}}}$ AND $E_{\mu_{\mathrm{s}}^{\prime}}$ FOR THE MAP EsTIMATES OF $\mu_{\mathrm{a}}$ AND $\mu_{\mathrm{s}}^{\prime}$ OBTAINED IN 2D FOR THE THREE DIFFERENT ILLUMINATION PAIRS. The Acoustic Sensors Were Located on Two Sides of THE TARGET: $\Omega_{\mathrm{L}}$ AND $\Omega_{\mathrm{B}}$

\begin{tabular}{ccccccc}
\hline & \multicolumn{3}{c}{$E_{\mu_{\mathrm{a}}}(\%)$} & \multicolumn{3}{c}{$E_{\mu_{\mathrm{s}}^{\prime}}(\%)$} \\
Noise levels $\epsilon$ & $5 \%$ & $1 \%$ & $0.1 \%$ & $5 \%$ & $1 \%$ & $0.1 \%$ \\
\hline \hline$s_{\mathrm{LR}}^{2 \mathrm{D}}$ and $s_{\mathrm{BT}}^{2 \mathrm{D}}$ & 22.3 & 7.9 & 4.1 & 9.3 & 7.2 & 4.8 \\
$s_{\mathrm{LB}}^{2 \mathrm{D}}$ and $s_{\mathrm{T}}^{2 \mathrm{D}}$ & 50.8 & 17.3 & 6.5 & 23.6 & 10.3 & 7.7 \\
$s_{\mathrm{L}}^{2 \mathrm{D}}$ and $s_{\mathrm{B}}^{2 \mathrm{D}}$ & 79.3 & 55.4 & 15.0 & 31.4 & 15.6 & 8.7 \\
\hline
\end{tabular}

\section{DISCUSSION AND CONCLUSION}

In this work, a Bayesian approach to the direct estimation of the optical parameters from photoacoustic time series was described. The forward model was based on coupling the optical and the acoustic propagation models where the DA was used for the optical propagation and the acoustic model was formed by using Green's function of the homogeneous wave equation. The MAP estimates of the optical absorption and scattering distributions were computed.

The numerical 2D simulations show, that when the acoustic view of the imaged domain becomes limited, the estimates of the optical parameters degrade in terms of relative error. This was shown with simulations where acoustic sensors were located on four, three, two and one edge of the imaged square shaped domain. Similar effect was seen when the sensors were located on either a circle or a semicircle. Qualitatively (visually) the estimates of the optical parameters were good in each sensor setting. In 2D simulations, it was found that the estimates can be improved if the noise level of the data is reduced, or if the sensors are placed such that they enclose the imaged domain at least partially. This suggests that the quality requirements on measurement systems with a limited view acoustic sensor geometry are more demanding than in settings where the acoustic measurement can be done in more enclosing fashion. In 3D, one numerical simulation was performed with the acoustic sensors located on one face of a cube shaped imaged target. Although the relative errors of the estimated optical parameters were higher, in comparison to the similar setting in 2D, the estimates were qualitatively good with the distinct shape and quantitative value of the estimated parameters being similar to the true parameters.
The work presented in this paper takes quantitative photoacoustic tomography closer to being practically realizable imaging modality. In comparison previously published similar approaches that estimate the optical properties directly form the photoacoustic time series [68]-[73], this work combines the full nonlinear photoacoustic forward model into a Bayesian inverse problem approach, providing estimates of both the optical absorption and scattering in acoustically limited view measurement setting in three spatial dimensions. The approach enables inclusion of sophisticated prior models of the parameters of interest and statistical information on the noise and uncertainties related to the imaging modality into the estimation process. In addition, the approach is able to provide posterior error intervals to aid assessing the quality of the estimates.

In this work the numerical implementation of the photoacoustic model, and the Bayesian approach used to form the estimates of the optical parameters, was done in MATLAB (R2011b, The MathWorks Inc., Natick, MA). Time to form the MAP estimates in 2D was within few minutes for a workstation equipped with Xeon E5649 (Intel Corporation, Santa Clara, CA) processor operating at $2.53 \mathrm{GHz}$, whereas in 3D the estimation took few days on a computer with Xeon E5-2630 operating at $2.40 \mathrm{GHz}$ (Intel Corporation, Santa Clara, CA). Increase in computational time, when shifting from $2 \mathrm{D}$ to $3 \mathrm{D}$, is expected as the number of unknowns grows exponentially with the number of dimensions. Part of the large difference between the estimation times is explained due code level differences: some of the optimizations done in 2D (e.g. precomputation of some of the matrices) could not be performed in 3D due to memory limitations. Part of the reason why the memory consumption was an issue in 3D is explained by the use of direct (and possibly) naive form of implementing the Gauss-Newton algorithm: this involved explicitly assembling large matrices and then using direct approaches to solve for Gauss-Newton iteration direction. Additional work needs to be done, in order to make the estimation process faster.

The current study was limited to acoustically homogeneous and non-attenuating medium, however, in practice the assumptions are not necessarily valid and imaged targets are likely to be acoustically more complex. Thus, the presented approach could be taken forward by using more complete acoustic simulation models. On the other hand, the DA was used as the optical simulation model in this work. A more realistic (as well as numerically more challenging) photoacoustic simulation model would be based on the RTE [39]-[41], [43]-[45], [67]. The use of the RTE would make it possible to investigate the parameter estimation problem of QPAT, for example, when the optical scattering has anisotropic behaviour, or when highly directed light sources are used. Recently, a direct estimation of the optical absorption from the photoacoustic time series using a Tikhonov regularized least squares scheme and the RTE as the forward model has been investigated [72].

The Bayesian approach, used in this work, makes it possible to incorporate quantitative prior information into the inverse problem. The approach also enables modelling of complex error sources, such as might be arising from physical acoustic 
sensors or light sources. These issues could be handled by using, for example, the approximation error method [74], [85], which has been used in QPAT in [51], [55].

In conclusion, the numerical results shown in this work demonstrate that by coupling the optical and acoustic simulation models to a single photoacoustic model, it is possible to estimate the optical parameters from the photoacoustic time series even in a acoustically limited view setting. The results thus indicate that achieving quantitative photoacoustic tomography should be feasible in practical acoustic measurement geometries.

\section{APPENDICES}

\section{APPENDIX [FEASIBILITY OF THE NORMAL APPROXIMATION OF THE POSTERIOR]}

When approximating a (posterior) density $\pi(x \mid y)$ with a (normal) distribution $\pi_{*}(x \mid y)$, s.t. $x \mid y \sim \mathcal{N}(\eta, \Gamma)$, the probabilities of events are generally never equal. Here, we define a safe approximation as one for which estimated errors based on the approximative marginal distributions are larger than those based on the actual posterior model. Thus, for example, we call the model safe if

$$
\int_{\eta_{i}-\chi \sigma_{i}}^{\eta_{i}+\chi \sigma_{i}} \pi_{*}\left(x_{i} \mid y\right) \mathrm{d} x_{i} \geq \int_{\eta_{i}-\chi \sigma_{i}}^{\eta_{i}+\chi \sigma_{i}} \pi\left(x_{i} \mid y\right) \mathrm{d} x_{i},
$$

for all $i=1, \ldots, 2 Q$, and some $\chi>0$, where $\sigma_{i}=\sqrt{\Gamma(i, i)}$. We note that for nonlinear models, the asymptotic decay of the actual posterior is often slower than for a normal model and thus the condition (36) can fail for a large enough $\chi$. The feasibility of the approximation depends on the underlying problem and the relevance of the choice of $\chi$, or the choice of the error interval in general.

For normal approximations, the probabilities of parameter being within $\pm \chi \sigma$ intervals corresponding to $\chi=1,2,3$ are $68.3 \%, 95.5 \%$, and $99.7 \%$ respectively. In order to compare the probabilities of the approximative posterior distribution derived error intervals to the normal distribution, a Monte Carlo approach is adopted.

Random samples of optical absorption $\mu_{\mathrm{a}}$ and $\mu_{\mathrm{s}}^{\prime}$, reminiscent to the parameter distribution studied in the main text, were generated, denoted by $x^{\ell}, \ell=1, \ldots, 100$. For each sample, the corresponding noisy data $y^{\ell}$ was simulated using the observation model (19) with the same noise levels $(5 \%, 1 \%$, and $0.1 \%)$, illuminations $\left(s_{\mathrm{LR}}^{2 \mathrm{D}}\right.$ and $\left.s_{\mathrm{BT}}^{2 \mathrm{D}}\right)$, and acoustic detection setup (detectors on two sides of the target), as used in results of Figure 4. The MAP estimates $x_{\text {MAP }}^{\ell}$, and the normal distribution statistics $\eta^{\ell}$ and $\Gamma^{\ell}$ of the approximative posterior distribution, were computed using the approach presented in Section III for each of the data $y^{\ell}$. From the covariance matrices $\Gamma^{\ell}$, standard deviations $\sigma^{\ell}$ were computed by taking the square root of the diagonal part of the matrices. Figure 7 shows a typical generated parameter distributions, their MAP estimates, and the $\pm 3 \sigma^{\ell}$ error intervals.

Based on the estimates $x_{\mathrm{MAP}}^{\ell}$, the true parameters $x^{\ell}$, and the standard deviations $\sigma^{\ell}$, sample based probability of $x^{\ell}$ being within the error interval $\left[x_{\mathrm{MAP}}^{\ell}-\chi \sigma^{\ell}, x_{\mathrm{MAP}}^{\ell}+\chi \sigma^{\ell}\right]$ was computed for $\chi=1,2,3$. The probabilities were computed
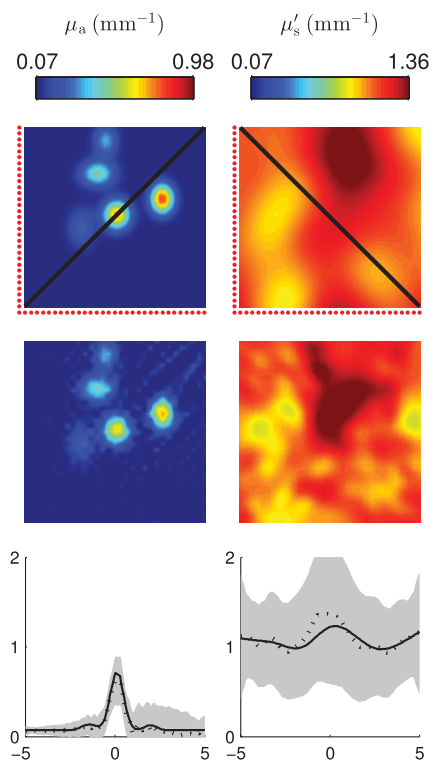

Fig. 7. An example of generated true parameters (top row), MAP estimates (second row), and approximative $\pm 3 \sigma$ posterior error intervals (bottom row) used in the analysis presented in the Appendix. The points at which the error intervals are shown are on diagonal cross sections across the domain and are indicated as black lines in the top row images. The error interval plots show the true parameters (solid line), the MAP estimates (dotted line), and the error intervals (grey areas). Four illuminating sides were used $\left(s_{\mathrm{LR}}^{2 \mathrm{D}}\right.$ and $\left.s_{\mathrm{BT}}^{2 \mathrm{D}}\right)$. The locations of acoustic sensors are indicated with the red dots. Results shown for $1 \%$ noise level.

TABLE IV

EVALUATION OF VALIDITY OF NORMal Distribution APPROXimation of Posterior Distribution. Shown Are SAmple Based ProbaBILITIES OF ESTIMATES OF $\mu_{\mathrm{a}}$ AND $\mu_{\mathrm{s}}^{\prime}$ BEING WITHIN $\chi$ STANDARD DEVIATIONS FROM THE TRUE PARAMETERS FOR NOISE LEVELS $5 \%, 1 \%$, AND $0.1 \%$. REFERENCE PROBABILITY, THAT A True Normal Distribution Has, Is Also Shown

\begin{tabular}{rcccc}
\hline & & $\chi=1$ & $\chi=2$ & $\chi=3$ \\
\hline \multirow{2}{*}{ Noise level 5\% } & $\mu_{\mathrm{a}}$ & $87.8 \%$ & $99.6 \%$ & $100.0 \%$ \\
& $\mu_{\mathrm{s}}^{\prime}$ & $94.5 \%$ & $100.0 \%$ & $100.0 \%$ \\
\hline \multirow{2}{*}{ Noise level 1\% } & $\mu_{\mathrm{a}}$ & $80.3 \%$ & $98.5 \%$ & $100.0 \%$ \\
& $\mu_{\mathrm{s}}^{\prime}$ & $98.2 \%$ & $100.0 \%$ & $100.0 \%$ \\
\hline \multirow{2}{*}{ Noise level 0.1\% } & $\mu_{\mathrm{a}}$ & $67.4 \%$ & $94.3 \%$ & $99.2 \%$ \\
& $\mu_{\mathrm{s}}^{\prime}$ & $98.5 \%$ & $100.0 \%$ & $100.0 \%$ \\
\hline \hline Reference & & $68.3 \%$ & $95.5 \%$ & $99.7 \%$ \\
\hline
\end{tabular}

by counting the proportion of discretization points, for which the true parameter was within $\chi$ standard deviations of the estimate, with respect to number of disretization points, for all $\ell$, and then taking the average of the proportions. The probabilities were computed for $\mu_{\mathrm{a}}$ and $\mu_{\mathrm{s}}^{\prime}$ separately. The sample based probabilities are shown in Table IV.

Based on the sample based probabilities, the normal distribution approximation of the posterior distribution, and the error intervals derived from the approximation, can be considered safe for both $\mu_{\mathrm{a}}$ and $\mu_{\mathrm{s}}^{\prime}$, except when the noise is small. The reason that the approximation fails at small noise levels, is likely to be caused by not including modelling errors in the observation model (19): at small error levels, the numerical errors of the forward model should be considered as well. However, even when the noise level is as low as $0.1 \%$, the error 
intervals derived from the approximate posterior statistics, are less than $1.2 \%$ away from being safe estimates of error for $\mu_{\mathrm{a}}$. Thus, the normal distribution approximation of the posterior distribution, and the error intervals derived from it, can be considered safe.

\section{REFERENCES}

[1] L. V. Wang, "Prospects of photoacoustic tomography," Med. Phys. vol. 35 , no. 12 , pp. 5758-5767, 2008.

[2] C. Li and L. V. Wang, "Photoacoustic tomography and sensing in biomedicine," Phys. Med. Biol., vol. 54, no. 19, pp. R59-R97, 2009.

[3] P. Beard, "Biomedical photoacoustic imaging," Interface Focus, vol. 5, no. 1 , pp. 602-631, 2011

[4] J. Xia, J. Yao, and L. V. Wang, "Photoacoustic tomography: Principles and advances," Prog. Electromagn. Res., vol. 147, pp. 1-22, 2014.

[5] M. Xu and L. V. Wang, "Universal back-projection algorithm for photoacoustic computed tomography," Phys. Rev. E, vol. 71, no. 1, p. 016706, 2005.

[6] P. Burgholzer, G. J. Matt, M. Haltmeier, and G. Paltauf, "Exact and approximative imaging methods for photoacoustic tomography using an arbitrary detection surface," Phys. Rev. E, vol. 75, no. 4, p. 046706, 2007.

[7] A. Rosenthal, D. Razansky, and V. Ntziachristos, "Fast semianalytical model-based acoustic inversion for quantitative optoacoustic tomography," IEEE Trans. Med. Imag., vol. 29, no. 6, pp. 1275-1285, Jun. 2010

[8] K. Wang, R. Su, A. A. Oraevsky, and M. A. Anastasio, "Investigation of iterative image reconstruction in three-dimensional optoacoustic tomography," Phys. Med. Biol., vol. 57, no. 17, pp. 5399-5423, 2012.

[9] M. Haltmeier, T. Schuster, and O. Scherzer, "Filtered backprojection for thermoacoustic computed tomography in spherical geometry," Math. Methods Appl. Sci., vol. 28, no. 16, pp. 1919-1937, 2005.

[10] L. A. Kunyansky, "Explicit inversion formulae for the spherical mean Radon transform," Inverse Problems, vol. 23, no. 1, pp. 373-383, 2007.

[11] L. A. Kunyansky, "Thermoacoustic tomography with detectors on an open curve: An efficient reconstruction algorithm," Inverse Problems, vol. 24 , no. 5 , p. 055021,2008

[12] D. V. Finch and Rakesh, "The spherical mean value operator with centers on a sphere," Inverse Problems, vol. 23, no. 6, pp. S37-S49, 2007.

[13] C. Zhang and Y. Wang, "Deconvolution reconstruction of full-view and limited-view photoacoustic tomography: A simulation study," J. Opt. Soc. Am. A, vol. 25, no. 10, pp. 2436-2443, 2008.

[14] L. Kunyansky, "Reconstruction of a function from its spherical (circular) means with the centers lying on the surface of certain polygons and polyhedra," Inverse Problems, vol. 27, no. 2, p. 025012, 2011.

[15] K. Wang and M. A. Anastasio, "A simple Fourier transform-based reconstruction formula for photoacoustic computed tomography with a circular or spherical measurement geometry," Phys. Med. Biol., vol. 57 , no. 23, pp. N493-N499, 2012.

[16] M. A. Anastasio, J. Zhang, D. Modgil, and P. J. La Rivière, "Application of inverse source concepts to photoacoustic tomography," Inverse Problems, vol. 23, no. 6, p. S21-35, 2007.

[17] F. Natterer, "Photo-acoustic inversion in convex domains," Inverse Problems Imag., vol. 6, no. 2, pp. 315-320, 2012.

[18] M. Agranovsky and P. Kuchment, "Uniqueness of reconstruction and an inversion procedure for thermoacoustic and photoacoustic tomography with variable sound speed," Inverse Problems, vol. 23, no. 5, pp. 2089-2102, 2007.

[19] Y. Hristova, P. Kuchment, and L. Nguyen, "Reconstruction and time reversal in thermoacoustic tomography in acoustically homogeneous and inhomogeneous media," Inverse Problems, vol. 24, no. 5, p. 055006, 2008.

[20] P. Stefanov and G. Uhlmann, "Thermoacoustic tomography with variable sound speed," Inverse Problems, vol. 25, no. 7, p. 075011, 2009.

[21] J. Qian, P. Stefanov, G. Uhlmann, and H. Zhao, "A new numerical algorithm for thermoacoustic and photoacoustic tomography with variable sound speed," Dept. Comput. Appl. Math., Univ. California, Los Angeles, Los Angeles, CA, USA, Tech. Rep. 10-81, 2010.

[22] B. T. Cox and B. E. Treeby, "Artifact trapping during time reversal photoacoustic imaging for acoustically heterogeneous media," IEEE Trans. Med. Imag., vol. 29, no. 2, pp. 387-396, Feb. 2010.

[23] J. Joseet al., "Speed-of-sound compensated photoacoustic tomography for accurate imaging," Med. Phys., vol. 39, no. 12, pp. 7262-7271, 2012.
[24] B. E. Treeby, E. Z. Zhang, and B. T. Cox, "Photoacoustic tomography in absorbing acoustic media using time reversal," Inverse Problems, vol. 26, no. 11, p. 115003, 2010.

[25] H. Roitner and P. Burgholzer, "Efficient modeling and compensation of ultrasound attenuation losses in photoacoustic imaging," Inverse Problems, vol. 27, no. 1, p. 015003, 2011.

[26] B. E. Treeby and B. T. Cox, “A k-space Green's function solution for acoustic initial value problems in homogeneous media with power law absorption," J. Acoust. Soc. Am., vol. 129, no. 6, pp. 3652-3660, 2011.

[27] Y. Xu, L. V. Wang, G. Ambartsoumian, and P. Kuchment, "Reconstructions in limited-view thermoacoustic tomography," Med. Phys., vol. 31, no. 4, pp. 724-733, 2004

[28] M. A. Anastasioet al., "Improving limited-view reconstruction in photoacoustic tomography by incorporatinga priori boundary information," Proc. SPIE, vol. 6856, p. 68561B, Feb. 2008.

[29] C. Huang, A. A. Oraevsky, and M. A. Anastasio, "Investigation of limited-view image reconstruction in optoacoustic tomography employing a priori structural information," Proc. SPIE, vol. 7800, p. 780004, Aug. 2010.

[30] A. Buehleret al., "Model-based optoacoustic inversions with incomplete projection data," Med. Phys., vol. 38, no. 3, pp. 1694-1704, 2011.

[31] B. T. Cox, S. R. Arridge, and P. C. Beard, "Photoacoustic tomography with a limited-aperture planar sensor and a reverberant cavity," Inverse Problems, vol. 23, no. 6, p. S95-S112, 2007.

[32] D. Wu, X. Wang, C. Tao, and X. J. Liu, "Limited-view photoacoustic tomography utilizing backscatterers as virtual transducers," Appl. Phys. Lett., vol. 99, no. 24, p. 244102, 2011.

[33] B. Huang, J. Xia, K. Maslov, and L. V. Wang, "Improving limited-view photoacoustic tomography with an acoustic reflector," J. Biomed. Opt., vol. 18, no. 11, p. 110505, 2013.

[34] G. Liet al., "Tripling the detection view of high-frequency linear-arraybased photoacoustic computed tomography by using two planar acoustic reflectors," Quant. Imag. Med. Surg., vol. 5, no. 1, pp. 57-62, 2015.

[35] P. Kuchment and L. Kunyansky, "Mathematics of photoacoustic and thermoacoustic tomography," in Handbook of Mathematical Methods in Imaging, New York: SpringerO. Scherzer, Ed., 2011, pp. 817-868.

[36] B. T. Cox, J. G. Laufer, and P. C. Beard, "The challenges for quantitative photoacoustic imaging," Proc. SPIE, vol. 7177, p. 717713, Feb. 2009.

[37] B. Cox, J. G. Laufer, S. R. Arridge, and P. C. Beard, "Quantitative spectroscopic photoacoustic imaging: A review," J. Biomed. Opt., vol. 17, no. 6, p. 061202, 2012.

[38] P. Mohajerani, S. Tzoumas, A. Rosenthal, and V. Ntziachristos, "Optical and optoacoustic model-based tomography: Theory and current challenges for deep tissue imaging of optical contrast," IEEE Signal Process. Mag., vol. 32, no. 1, pp. 88-100, Jan. 2015.

[39] L. Yao, Y. Sun, and H. Jiang, "Transport-based quantitative photoacoustic tomography: Simulations and experiments," Phys. Med. Biol., vol. 55, no. 7, pp. 1917-1934, 2010.

[40] B. Cox, T. Tarvainen, and S. Arridge, "Multiple illumination quantitative photoacoustic tomography using transport and diffusion models," in Tomography and Inverse Transport Theory (Contemporary Mathematics), vol. 559, G. Bal, D. Finch, P. A. Kuchment, J. Schotland, P. Stefanov and G. Uhlmann, Eds. Providence, RI, USA: AMS, 2011.

[41] T. Tarvainen, B. T. Cox, J. P. Kaipio, and S. R. Arridge, "Reconstructing absorption and scattering distributions in quantitative photoacoustic tomography," Inverse Problems, vol. 28, no. 8, p. 084009, 2012.

[42] X. Li and H. Jiang, "Impact of inhomogeneous optical scattering coefficient distribution on recovery of optical absorption coefficient maps using tomographic photoacoustic data," Phys. Med. Biol., vol. 58, no. 4, pp. 999-1011, 2013.

[43] T. Saratoon, T. Tarvainen, B. T. Cox, and S. R. Arridge, "A gradientbased method for quantitative photoacoustic tomography using the radiative transfer equation," Inverse Problems, vol. 29, no. 7, p. 075006 , 2013.

[44] A. V. Mamonov and K. Ren, "Quantitative photoacoustic imaging in radiative transport regime," Commun. Math. Sci., vol. 12, no. 2, pp. 201-234, 2014.

[45] A. Pulkkinen, B. T. Cox, S. R. Arridge, J. P. Kaipio, and T. Tarvainen, "Quantitative photoacoustic tomography using illuminations from a single direction," J. Biomed. Opt., vol. 20, no. 3, p. 036015, 2015.

[46] B. T. Cox, S. R. Arridge, K. P. Köstli, and P. C. Beard, "Two-dimensional quantitative photoacoustic image reconstruction of absorption distributions in scattering media by use of a simple iterative method," Appl. Opt., vol. 45, no. 8, pp. 1866-1875, 2006. 
[47] L. Yin, Q. Wang, Q. Zhang, and H. Jiang, "Tomographic imaging of absolute optical absorption coefficient in turbid media using combined photoacoustic and diffusing light measurements," Opt. Lett., vol. 32, no. 17, pp. 2556-2558, 2007.

[48] B. Banerjee, S. Bagchi, R. M. Vasu, and D. Roy, "Quantitative photoacoustic tomography from boundary pressure measurements: Noniterative recovery of optical absorption coefficient from the reconstructed absorbed energy map," J. Opt. Soc. Am. A, vol. 25, no. 9, pp. 2347-2356, 2008.

[49] R. J. Zemp, "Quantitative photoacoustic tomography with multiple optical sources," Appl. Opt., vol. 49, no. 18, pp. 3566-3572, 2010.

[50] H. Gao, H. Zhao, and S. Osher, "Quantitative photoacoustic tomography," Dept. Comput. Appl. Math., Univ. California, Los Angeles, Los Angeles, CA, USA, Tech. Rep. 11-28, 2011.

[51] T. Tarvainen, A. Pulkkinen, B. T. Cox, J. P. Kaipio, and S. R. Arridge, "Bayesian image reconstruction in quantitative photoacoustic tomography," IEEE Trans. Med. Imag., vol. 32, no. 12 , pp. 2287-2298, Dec. 2013

[52] W. Naetar and O. Scherzer, "Quantitative photoacoustic tomography with piecewise constant material parameters," SIAM J. Imag. Sci., vol. 7, no. 3, pp. 1755-1774, 2014.

[53] P. Shao, T. J. Harrison, and R. J. Zemp, "Consecutively reconstructing absorption and scattering distributions in turbid media with multipleillumination photoacoustic tomography," J. Biomed. Opt., vol. 19, no. 12, p. 126009, 2014.

[54] X. Zhang, W. Zhou, X. Zhang, and H. Gao, "Forward-backward splitting method for quantitative photoacoustic tomography," Inverse Problems, vol. 30, no. 12, p. 125012, 2014.

[55] A. Pulkkinenet al., "Approximate marginalization of unknown scattering in quantitative photoacoustic tomography," Inverse Problems Imag., vol. 8, no. 3, pp. 811-829, 2014.

[56] A. Q. Bauer, R. E. Nothdurft, T. N. Erpelding, L. V. Wang, and J. P. Culver, "Quantitative photoacoustic imaging: Correcting for heterogeneous light fluence distributions using diffuse optical tomography," J. Biomed. Opt., vol. 16, no. 9, p. 096016, 2011.

[57] K. Ren, H. Gao, and H. Zhao, "A hybrid reconstruction method for quantitative PAT," SIAM J. Imag. Sci., vol. 6, no. 1, pp. 32-55, 2013.

[58] D. Razansky, C. Vinegoni, and V. Ntziachristos, "Multispectral photoacoustic imaging of fluorochromes in small animals," Opt. Lett., vol. 32, no. 19, pp. 2891-2893, 2007.

[59] Z. Yuan and H. Jiang, "Simultaneous recovery of tissue physiological and acoustic properties and the criteria for wavelength selection in multispectral photoacoustic tomography," Opt. Lett., vol. 34, no. 11, pp. 1714-1716, 2009.

[60] D. Razansky et al., "Multispectral opto-acoustic tomography of deepseated fluorescent proteins in vivo," Nature Photon., vol. 3, pp. 412-417, Jun. 2009

[61] B. T. Cox, S. R. Arridge, and P. C. Beard, "Estimating chromophore distributions from multiwavelength photoacoustic images," J. Opt. Soc. Am. A, vol. 26, no. 2, pp. 443-455, 2009.

[62] J. Laufer, B. Cox, E. Zhang, and P. Beard, "Quantitative determination of chromophore concentrations from 2D photoacoustic images using a nonlinear model-based inversion scheme," Appl. Opt., vol. 49, no. 8, pp. 1219-1233, 2010.

[63] A. Pulkkinen, B. T. Cox, S. R. Arridge, J. P. Kaipio, and T. Tarvainen, "A Bayesian approach to spectral quantitative photoacoustic tomography," Inverse Problems, vol. 30, no. 6, p. 065012, 2014.
[64] G. Bal and K. Ren, "Multi-source quantitative photoacoustic tomography in a diffusive regime," Inverse Problems, vol. 27, no. 7, p. 075003 , 2011.

[65] G. Bal and K. Ren, "On multi-spectral quantitative photoacoustic tomography in diffusive regime," Inverse Problems, vol. 28, no. 2, p. 025010 , 2012.

[66] G. Bal and G. Uhlmann, "Inverse diffusion theory of photoacoustics," Inverse Problems, vol. 26, no. 8, p. 085010, 2010.

[67] G. Bal, A. Jollivet, and V. Jugnon, "Inverse transport theory of photoacoustics," Inverse Problems, vol. 26, no. 2, p. 025011, 2010.

[68] P. Shao, T. Harrison, and R. J. Zemp, "Iterative algorithm for multiple illumination photoacoustic tomography (MIPAT) using ultrasound channel data," Biomed. Opt. Exp., vol. 3, no. 12, pp. 3240-3249, 2012.

[69] N. Song, C. Deumié, and A. Da Silva, "Considering sources and detectors distributions for quantitative photoacoustic tomography," Biomed. Opt. Exp., vol. 5, no. 11, pp. 3960-3974, 2014.

[70] T. Ding, K. Ren, and S. Vallélian, "A one-step reconstruction algorithm for quantitative photoacoustic imaging," Inverse Problems, vol. 31, no. 9 , p. 095005, 2015.

[71] Z. Yuan and H. Jiang, "A calibration-free, one-step method for quantitative photoacoustic tomography," Med. Phys., vol. 39, no. 11, pp. 6895-6899, 2012.

[72] M. Haltmeier, L. Neumann, and S. Rabanser, "Single-stage reconstruction algorithm for quantitative photoacoustic tomography," Inverse Problems, vol. 31, no. 6, p. 065005, 2015.

[73] H. Gao, J. Feng, and L. Song, "Limited-view multi-source quantitative photoacoustic tomography," Inverse Problems, vol. 31, no. 6, p. 065004 , 2015.

[74] J. P. Kaipio and E. Somersalo, Statistical and Computational Inverse Problems. New York: Springer, 2005.

[75] A. Tarantola, Inverse Problem Theory and Methods for Model Parameter Estimation. Philadelphia, PA, USA: SIAM, 2005.

[76] D. Calvetti and E. Somersalo, Introduction to Bayesian Scientific Computing: Ten Lectures on Subjective Computing. New York: Springer 2007.

[77] A. Ishimaru, Wave Propagation and Scattering in Random Media, vol. 1. San Francisco, CA, USA: Academic, 1978.

[78] P. G. Ciarlet, The Finite Element Method for Elliptic Problems. Philadelphia, PA, USA: SIAM, 2002.

[79] B. T. Cox and P. C. Beard, "Fast calculation of pulsed photoacoustic fields in fluids using k-space methods," J. Acoust. Soc. Am., vol. 117 no. 6, pp. 3616-3627, 2005.

[80] V. Z. Gusev and A. A. Karabutov, Laser Optoacoustics. College Park, MD, USA: AIP, 1992.

[81] D. G. Duffy, Green's Functions With Applications. Boca Raton, FL, USA: Chapman \& Hall, 2001.

[82] J. Nocedal and S. J. Wright, Numerical Optimization. New York: Springer, 1999.

[83] C. E. Rasmussen and C. K. I. Williams, Gaussian Processes for Machine Learning. Cambridge, MA, USA: MIT Press, 2006.

[84] W. F. Cheong, S. A. Prahl, and A. J. Welch, "A review of the optical properties of biological tissues," IEEE J. Quant. Electron., vol. 26 , no. 12, pp. 2166-2185, Dec. 1990.

[85] J. Kaipio and E. Somersalo, "Statistical inverse problems: Discretization, model reduction and inverse crimes," J. Comput. Appl. Math., vol. 198, no. 2, pp. 493-504, 2007. 